\section{Turning lupus against cancer}

\section{By Lauren Martz, Staff Writer}

Yale School of Medicine researchers have shown that a lupus autoantibody inhibited DNA repair mechanisms in cancer cells and sensitized mice to radiation and chemotherapy. ${ }^{1}$ The group is planning Phase I trials of the autoantibody in patients with cancer.

Although DNA damage repair responses are necessary to combat the toxic effects of environmental and chemical stressors, the responses can reduce or block the efficacy of chemotherapy and radiation therapies.

To increase the efficacy of chemotherapy and radiation, researchers have begun to identify compounds that inhibit the DNA damage repair response in cancer cells and could be delivered as adjuncts to chemotherapy. One approach in the clinic is inhibition of poly(ADP-ribose) polymerase (PARP), which is an upstream component of a single-strand DNA break repair pathway. Inhibition of PARP sensitizes cancers to DNAdamaging therapies but only reduces activity of the single-strand DNA break repair pathway. At least eight companies have PARP inhibitors in clinical testing to treat various cancers.

Now, Peter Glazer and colleagues at Yale have shown that a lupus autoantibody blocks the DNA damage repair response in cancer cells.

Glazer is chairman of the Department
"[The 3E10 autoantibody] inhibits two important DNA repair pathways that many cancers depend on to resist chemotherapy and radiation. Existing therapies, such as PARP inhibitors, inhibit just one of these pathways, so the antibody has the potential to be more effective than PARP inhibitors in some settings."

- Peter Glazer, Yale School of Medicine
Subsequent studies found that the antibody entered cells via interactions between its single-chain variable fragment $(\mathrm{scFv})$ and the equilibrative nucleoside transporter 2 (ENT2) surface receptor. ${ }^{3}$

Building on those previous studies, Glazer and colleagues set out to use $3 \mathrm{E} 10$ as a delivery vehicle that enhances the radioprotective effect of heat shock protein 70 (Hsp70) in breast cancer cells. However, the team found that 3E10 alone was sufficient to improve the effects of radiation.

In addition, both breast cancer and glioma cells had greater sensitivity to the DNA-damaging chemotherapeutic doxorubicin when reated with $3 \mathrm{E} 10$.

In mice with human glioma xenografts, $3 \mathrm{E} 10$ plus doxorubicin or radiation increased tumor suppressive effects compared with xorubicin or radiation alone. against tumors with intrinsic deficiencies in DNA damage responses, such as breast, ovarian and prostate cancers with mutations in breast cancer 2 early onset (BRCA2).

Indeed, $3 \mathrm{E} 10$ alone triggered cell death in BRCA2-deficient human ovarian cancer cells but not in BRCA2proficient cells. $3 \mathrm{E} 10$ plus doxorubicin or radiation further improved the anticancer effect in BRCA2-deficient ovarian or prostate cancer cells.

The findings were published in Science Translational Medicine.

The 3E10 autoantibody "inhibits two important DNA repair pathways that many cancers depend on to resist chemotherapy and radiation. Existing therapies, such as PARP inhibitors, inhibit just one of these pathways, so the antibody has the potential to be more effective than PARP inhibitors in some settings," said corresponding author Glazer.

The paper also included researchers from the VA Greater Los Angeles Healthcare System, the Beckman Research Institute at City of Hope, the University of California, Los Angeles and The University of Vermont. Medicine.

In 1999, researchers at the University Hospital of Lausanne tested a DNA-targeting lupus autoantibody, 3E10, that was identified in a mouse model of systemic lupus erythematosus (SLE) as a vaccine in patients with SLE. ${ }^{2}$ The team found that the antibody was safe, but they dropped development because of competing priorities and because it crossed the cell membrane and entered into the nucleus, which could cause negative effects on cellular gene expression.

Francois Spertini, associate professor and internist in the Department of Immunology and Allergy at the University Hospital of Lausanne and lead author on the 1999 study, told SciBX that his team did not pursue $3 \mathrm{E} 10$ further due to competing priorities, although the vaccine was immunogenic. Despite potential risks of nuclear penetration, his team did not see any related safety issues in the patients with SLE.

\section{Clinical safety}

Glazer told SciBX that his group's next steps "are to determine the impact of $3 \mathrm{E} 10$ on cancer cells with a range of deficiencies in DNA repair to determine the tumors that will be most sensitive to the antibody."

He plans to start Phase I testing of the antibody in two to three years in glioblastoma multiforme (GBM) and certain breast, ovarian and pancreatic tumors. "Our initial laboratory work has shown that the antibody is especially good at radiosensitizing malignant gliomas. Since the standard of care for high-grade gliomas is radiation therapy followed by surgical resection, we think that clinical testing in this setting would make sense."

Spertini said it is not yet clear from the mouse trials how often the 


\section{ANALYSIS}

antibody will need to be given. "The antibodies do not have a long half-life in vivo. We often treat autoimmune patients with monoclonal antibodies, and we need to repeat administration every month in most cases for a sustained effect."

"One issue with treating humans with this antibody is that it is a murine antibody, and it is possible that humans could develop neutralizing antibodies. In the human trial in 1999, it appears that 3E10 did induce significant neutralizing antibodies, suggesting that one might need to modify the antibody for prolonged human use," said Andrew Allen, EVP of clinical and preclinical development and CMO of Clovis Oncology Inc.

Clovis' rucaparib, an oral PARP inhibitor, is in Phase I testing to treat breast and ovarian cancers. The company also has a partnership with Foundation Medicine Inc. for a companion diagnostic that sequences the tumors to identify patients with $B R C A$ mutations or related mutations that would best benefit from the treatment.

Glazer countered that "it is not necessary to humanize the antibody if it will be used in cancer therapy regimens in one or a few short doses."

He added that the limited doses also prevent the risk of causing lupus. "For cancer therapy, the antibody would just be given a few times and would be administered transiently. To cause lupus, it would need to be consistently present in high amounts," he said.

Allen said another potential issue is that mice received a dose of the antibody that equates "to about $50 \mathrm{mg} / \mathrm{kg}$ in humans," which would likely be too expensive to manufacture.

He added, "The other problem with such high doses is that they can create toxicities by nonspecific binding."
Glazer told SciBX that his team is working to improve the potency of the antibody to reduce the doses required for efficacy. He also said commercial antibody production should lead to higher activity and purity than the antibodies used in the mouse studies.

Additionally, he said that "our preliminary work in BRCA2-deficient ovarian cancer cells suggests that low doses of the antibody may be very effective in such cells."

Glazer said the Yale School of Medicine has filed a patent application covering the work. The IP is available for licensing.

Martz, L. SciBX 5(44); doi:10.1038/scibx.2012.1151

Published online Nov. 8, 2012

\section{REFERENCES}

1. Hansen, J.E. et al. Sci. Transl. Med.; published online Oct. 24, 2012; doi:10.1126/scitranslmed.3004385

Contact: Peter M. Glazer, Yale School of Medicine, New Haven, Conn.

e-mail: peter.glazer@yale.edu

2. Spertini, F. et al. J. Rheumatol. 26, 2602-2608 (1999)

3. Lisi, S. et al. Clin. Exp. Med. 11, 1-10 (2011)

\section{COMPANIES AND INSTITUTIONS MENTIONED}

Beckman Research Institute at City of Hope, Duarte, Calif. Clovis Oncology Inc. (NASDAQ:CLVS), Boulder, Colo.

Foundation Medicine Inc., Cambridge, Mass.

University Hospital of Lausanne, Lausanne, Switzerland

University of California, Los Angeles, Calif.

The University of Vermont, Burlington, Vt.

VA Greater Los Angeles Healthcare System, Sepulveda, Calif. Yale School of Medicine, New Haven, Conn. 